\title{
A Model Comparison Approach to Posterior Predictive Model Checks in Bayesian Confirmatory Factor Analysis
}

Bayesian estimation for structural equation models (SEM) is a viable alternative to frequentist SEM approaches (e.g., maximum likelihood), particularly for complex model specifications or for analyses with small sample sizes (e.g. Muthén \& Asparouhov, 2012). The popularity of Bayesian approaches in SEM carries with it the need for Bayesian-based model fit investigations to examine global or local model misfit in Bayesian SEM analyses. Common types of SEM model misspecification include the omission of needed latent variables (e.g., Kaplan, 1988), the misspecification of which observed indicator variables measure which latent variable, or violations of latent variable distributional assumptions (e.g., Boomsma, 1987). Posterior predictive model checks (PPMCs) are tools used in Bayesian analyses to help detect model misspecifications by comparing statistics calculated from observed data with model-generated data based on the posterior estimates of parameters (e.g., Gelman et al., 1996, Levy, 2011; Levy et al., 2009). In this paper, we propose and investigate a novel method for investigating model fit in Bayesian CFA (and SEM) using model comparison PPMC (MC-PPMC).

In PPMCs, the value of an observed statistic is compared to the predictive distribution of the same statistic calculated from simulated data sets; these simulated data sets are generated from draws from the posterior distribution of model parameters. This comparison is facilitated by the percentile rank of the observed statistic of the posterior predictive distribution, oftent called the posterior predictive $p$-value (PPP value). The PPP value represents the location of an observed statistic relative to the posterior predicted distribution, which is different from an maximum likelihood (ML)-based p-value. The ML-based p-value is a quantity derived from the likelihood function or the limiting distribution of model parameters. PPP values close to zero or one (at the tails of posterior predictive distribution) normally indicate bad model-data fit. This concept was originated by Rubin and was later extended to include general discrepancies by Gelman et al. (1996). 
One concern in the use of PPP values is that the PPP value may be heavily influenced by the reference point from the observed data, which is often ML-based. For instance, when examining the local misfit between a pair of observed indicators in a Bayesian CFA model, the ML estimate of the Pearson correlation is commonly used as the observed statistic in a PPP analysis. In such analyses, the Pearson correlation between a pair of observed indicators is calculated using ML and then the percentile of that quantity is found using the predictive distribution of the correlation. Research on PPP values in latent variable modeling has been mixed, in some cases finding Type I error rates to be less than nominal values and in other cases finding Type I error rates at or slightly below nominal values (Levy, 2011). PPP values can also yield overly conservative results (Robins et al., 2000). Under small sample sizes, the empirical distribution of observed statistics used for calculating PPP values can be large, which may affect the accuracy of the PPP value calculation. Additionally, as the observed test statistics are ML-based point estimates, their realized values may depend on asymptotic arguments to be consistent while a posterior distribution (rather than a point estimate) does not have the same asymptotic dependency, at least in small samples (Levy, 2011).

Our proposed MC-PPMC replaces the ML-based point estimate from the observed data with the predictive distribution of a Bayesian-estimated saturated model, resulting in model fit being judged by the comparison between the two distributions. The saturated model in CFA assumes the observed data follow a multivariate normal distribution where all means, variances, and covariances are estimated. In ML-based CFA, the saturated model is the basis for model fit comparisons globally (i.e., likelihood ratio tests and Root Mean Squared Error of Approximation values) and locally (i.e., standardized, normalized, and unstandardized residual covariances). In our proposed method, we use a saturated model as a reference distribution, estimated with uninformative priors. Instead of using PPP values formed by comparing point estimates of statistics to their respective posterior predictive distributions, our method seeks to quantify a measure of overlap between the 
posterior predictive distributions of the saturated and specified models. When these posterior predictive distributions have a high degree of overlap, the specified model can be considered to fit the data well. Alternatively, when these posterior predictive distributions show little overlap, model fit of the specified model can be considered to be poor.

The remainder of the paper defines MC-PPMC and examines its use in both simulation and empirical data analyses. First, we introduce various types of model fit indices in either ML or Bayesian analyses. Next, we present MC-PPMC using the Kolmogorov-Smirnov (KS) statistic to quantify the degree of distributional overlap. To check the accuracy of the proposed measures, we describe the results of a simulation study, in which the performance of our proposed methods is compared with ML-constructed PPP values. We then apply our methods to an empirical example in order to show the performance of the proposed method in a real-world scenario. Finally, we discuss the advantages and the limitations of our new methods along with future extensions of these approaches.

\section{Confirmatory Factor Models}

Confirmatory factor analysis (CFA) models posit that vector of responses by a person $p(p=1, \ldots, N)$ to a set of observed indicator variables $i(i=1, \ldots, I)$, $\boldsymbol{Y}_{p}=\left[Y_{p 1}, \ldots, Y_{p I}\right]^{T}$, is influenced by the value of a set of $k=1, \ldots, K$ latent factors $\boldsymbol{\xi}_{p}=\left[\xi_{p 1}, \ldots, \xi_{p K}\right]$ via a multivariate linear model:

$$
\boldsymbol{Y}_{p}=\boldsymbol{\mu}+\Lambda \boldsymbol{\xi}_{p}+\boldsymbol{\delta}_{p}
$$

where $\boldsymbol{\Lambda}$ is an $I \times K$ matrix of factor loadings, $\boldsymbol{\mu}$ is an $I \times 1$ vector of item intercepts, and $\boldsymbol{\delta}$ is an $I \times 1$ vector of item-specific residuals. For model identification, a set constraints are placed on the elements of $\boldsymbol{\Lambda}$ where, $\lambda_{i k}=0$ if item $i$ does not measure factor $k$ (e.g., McDonald, 1999). The residuals are assumed to follow a multivariate normal distribution with zero mean vector and (often diagonal) covariance matrix $\Psi$. 
Factors are assumed to follow a multivariate normal distribution, often with mean vector fixed to zero and factor covariance matrix $\boldsymbol{\Phi}$. Additional constraints may be placed on the item intercepts and factor loadings so as to estimate the factor means and variances, respectively. Coupling the assumed distribution of the factors (with zero mean vector) and item residuals with the linear model in Equation 1 results in the assumption that the data follow a multivariate normal distribution with mean vector equal to item intercepts $\boldsymbol{\mu}$ and covariance matrix given by:

$$
\Sigma_{0}=\Lambda \boldsymbol{\Phi} \Lambda^{T}+\boldsymbol{\Psi} .
$$

The CFA model puts a specific hypothesized structure on the covariance matrix of the observed indicators. Prominently featured in CFA analyses is a comparison of the estimates of a hypothesized CFA model (which we label $H_{0}$ ) with covariance matrix $\boldsymbol{\Sigma}_{0}$ with that of a general, saturated model (which we label $H_{1}$ ) with no constraints on its covariance matrix $\Sigma_{1}$. As all possible CFA models are nested within the saturated model $H_{1}$, the comparison of these two models is conducted via mechanisms of nested model comparisons (such as likelihood ratio tests for ML-based analyses), where the CFA model with covariance matrix $\boldsymbol{\Sigma}_{0}$ represents the null (hypothesized) model $\left(H_{0}\right)$ and the saturated model with $\Sigma_{1}$ represents the alternative model $\left(H_{1}\right)$. In ML-based analyses, global fit statistics are derived from this comparison including the model Chi-Squared test and the root mean square error of approximation (RMSEA). Moreover, local model misfit is often conducted by inspection of the residual elements of the difference between $\boldsymbol{\Sigma}_{0}$ and $\boldsymbol{\Sigma}_{1}$ with raw, standardized, and normalized versions being used, the latter two involving estimates of the elements of unconstrained saturated model covariance matrix $\boldsymbol{\Sigma}_{1}$.

\section{Bayesian Confirmatory Factor Models}

In general, Bayesian estimation methods seek to find the posterior distribution of a set of parameters $\boldsymbol{\theta}_{H}$ for a hypothesized model $H$. This distribution is given by Bayes 
theorem in Equation (3):

$$
p\left(\boldsymbol{\theta}_{H} \mid \boldsymbol{Y}\right) \propto p\left(\boldsymbol{Y} \mid \boldsymbol{\theta}_{H}\right) p\left(\boldsymbol{\theta}_{H}\right),
$$

where $p\left(\boldsymbol{\theta}_{H}\right)$ is the prior distributions of the parameters and $p\left(\boldsymbol{Y} \mid \boldsymbol{\theta}_{H}\right)$ is the model likelihood function of the data given the parameters. In Bayesian CFA models, a conditional approach to estimation is frequently implemented where $p\left(\boldsymbol{Y} \mid \boldsymbol{\theta}_{H}\right)$ is given by the multivariate normal density with mean vector equal to $\boldsymbol{\mu}+\boldsymbol{\Lambda} \boldsymbol{\Xi}$ and covariance matrix $\Psi$. In such conditional models, an additional step is needed to specify the likelihood of the unobserved factors $\boldsymbol{\Xi}$, which is specified as multivariate normal with zero mean vector and covariance matrix $\boldsymbol{\Phi}$.

To estimate the Bayesian CFA model, prior distributions are specified for each type of parameter, specifically, the item intercepts $\boldsymbol{\mu}$, the factor loadings $\boldsymbol{\Lambda}$, the unique variances $\boldsymbol{\Psi}$, and the factor variances and covariances in $\boldsymbol{\Phi}$. The latent variables $\boldsymbol{\Xi}$ are treated as parameters that have a prior distribution equal to their assumed factor distribution, which is multivariate normal with mean zero and covariance matrix $\mathbf{\Phi}$. Although prior distributions can vary for each type of parameter, often the item intercepts and factor loadings follow normal distributions (which are conjugate priors, enabling direct sampling from the posterior distribution) and with unique variances following inverse gamma prior distributions (also conjugate priors). A conjugate prior for the factor covariance matrix is the inverse Wishart distribution.

\section{Bayesian Saturated Models}

In Bayesian statistics, the posterior distribution is obtained for all parameters - meaning the Bayesian version of the saturated model $\left(H_{1}\right)$ includes a set of prior distributions for the elements of the mean vector and elements of the covariance matrix $\Sigma_{1}$ and results in the construction of a posterior distribution for each unique parameter in the model. Herein lies a critical difference between the saturated model $H_{1}$ estimated using ML-based methods and Bayesian methods: ML-based methods use the 
ML-based point estimate for all parameters of the saturated model $H_{1}$ whereas the Bayesian analog of the saturated model $H_{1}$ necessarily has a posterior distribution for all parameters. Although the posterior distribution will converge in distribution to the ML asymptotic distribution as the sample size goes to infinity, wide variability may exist for cases where sample sizes are small relative to the number of parameters.

The key feature of ML-based PPP values this study seeks to investigate is how to incorporate variability of the saturated model's posterior distributions into the model fit process. That is, when the saturated model $H_{1}$ (sample) means, variances, and covariances yield posterior distributions rather than point estimates, how do model fit indices change? Moreover, does variability in the posterior distribution need to be accounted for when evaluating the model fit of the specified model $H_{0}$ ?

\section{Evaluation of Bayesian CFA Model Fit}

To illustrate Bayesian Structural Equation Modeling (BSEM) and Bayesian CFA fit indices, Levy (2011) distinguished two separate types of Bayesian model fit evaluations in terms of their target measures: test statistics and discrepancy measures. The first approach focuses on the extent to which the model recovers or predicts features of the data, whereas the second aims to explicitly build in the comparison between the observed data and model-implied data characteristics. Our proposed MC-PPMC method provides discrepancy measures that indicate areas of local model misfit.

An advantage of Bayesian SEM fit indices over most of their frequentist counterparts is that the posterior distribution allows uncertainty to be quantified for any index. Despite different processes, Bayesian and ML-based model fit methods have shown to have similar rejection rates when sample sizes are large. For example, Garnier-Villarreal and Jorgensen (2019) compared the the chi-square-based approximate fit indices that are commonly used in SEM to their Bayesian analogs through a simulation study and concludes that Markov Chain Monte Carlo (MCMC) with noninformative priors yields similar results to MLEs 
across varied levels of misspecification, sample sizes, and model types.

\section{Model Comparison Posterior Predictive Model Checking}

Common Bayesian PPMC methods work to find the posterior predictive distribution conditional of the parameters of the specified model $p\left(Y_{H}^{r e p} \mid \boldsymbol{\theta}_{H}\right)$ :

$$
p\left(\boldsymbol{Y}^{r e p} \mid \boldsymbol{\theta}_{H}\right)=\int_{\boldsymbol{\theta}_{H}} p\left(\boldsymbol{Y}^{r e p} \mid \boldsymbol{\theta}_{H}\right) p\left(\boldsymbol{\theta}_{H} \mid \boldsymbol{Y}^{\text {obs }}\right) d \boldsymbol{\theta}_{H} \propto \int_{\boldsymbol{\theta}_{H}} p\left(\boldsymbol{Y}^{r e p} \mid \boldsymbol{\theta}_{H}\right) p\left(Y^{o b s} \mid \boldsymbol{\theta}_{H}\right) p\left(\boldsymbol{\theta}_{H}\right) d \boldsymbol{\theta}_{H} .
$$

Equation (4) shows the general form of the posterior predictive distribution, $p\left(\boldsymbol{Y}^{\text {rep }} \mid \boldsymbol{\theta}_{H}\right)$, which is the integral of two components: the sampling distribution of the replicated data given the sampled values from the posterior distribution of parameters under model $H$, $p\left(\boldsymbol{Y}^{\text {rep }} \mid \boldsymbol{\theta}_{H}\right)$, and the posterior distribution of parameter under model $H, \mathrm{p}\left(\boldsymbol{\theta}_{\mathrm{H}} \mid \mathrm{Y}^{\text {obs }}\right)$.

In practice, PPMC methods are implemented by simulating data based on the model being estimated. To provide context, consider an example where a Bayesian CFA model of Equation (11) is estimated via MCMC. First, a standard MCMC estimation algorithm is run (with specifications of prior distributions, number of Markov chains, number of iterations, burn-in, etc.). Once the chains have been estimated and chain convergence is established, then a sample of parameters $\left(\boldsymbol{\theta}_{H}\right)$ are drawn with replacement from the set of iterations of the Markov chains. For each sampled set of parameters, a set of data $\left(\boldsymbol{Y}^{\text {rep }}\right)$ with sample size equal to that of the observed data are simulated by plugging the sampled parameters into the model $H$. Then, the test statistics $T\left(\boldsymbol{Y}^{r e p}\right)$ are calculated from the newly-generated data. In our case, $T(\cdot)$ will be the Pearson correlation coefficients calculated for each pair of observed indicators. Across all replication samples of the posterior distribution of parameters, $T\left(\boldsymbol{Y}^{r e p}\right)$ is calculated, yielding, for each pair of observed indicators, the predictive distribution of the statistic. The principle of PPMC is to locate the position of the observed data statistic $\mathrm{T}\left(\boldsymbol{Y}^{\mathrm{obs}}\right)$ in the posterior predictive distribution $p\left(\boldsymbol{Y}^{\text {rep }} \mid \boldsymbol{\theta}_{H}\right)$. The key difference between MC-PPMC and PPMC with classical ML-based fit indices lies in the choice of the reference distribution (e.g., Lee et al., 2016). 
Under standard PPMC methods, the reference distribution is the posterior predictive distribution, to which the often ML-based observed data statistic $\mathrm{T}\left(\mathrm{Y}^{\mathrm{obs}}\right)$ is compared.

As standard PPMC methods typically use ML-based observed data statistics $\mathrm{T}\left(\boldsymbol{Y}^{\text {obs }}\right)$, they do not incorporate uncertainty of the observed data statistics into the process. Such uncertainty of observed data statistics may come from sampling error which may especially be prevalent in situations where there are numerous cases of missing data or small sample sizes are present. Asparouhov and Muthén (2020) recently proposed a new approach by using the parameters of the saturated model $\left(H_{1}\right)$ to generate the posterior predictive distribution, which could reduce the rejection error rate in such situations.

The principal motivation of this study is to replace the point estimate of observed data statistics, $\mathrm{T}\left(\boldsymbol{Y}^{\text {obs }}\right)$, with the posterior predictive distribution of observed data statistics, $\mathrm{T}\left(\boldsymbol{Y}^{\mathrm{obs}} \mid \boldsymbol{\theta}_{\boldsymbol{H}}\right)$. This approach not only depicts the discrepancy between the observed data with reference distribution under the saturated model $H_{1}$ but also the degree of uncertainty in the observed data statistics.

\section{Proposed Model Comparison PPMC Procedure}

In order to get the posterior distribution of the covariance matrix $\Sigma_{1}$ from saturated model $H_{1}$, we use a Bayesian algorithm to estimate the saturated model using the observed data. Choices of prior distributions for the saturated model are critical as overly strict priors may result in saturated model posterior distributions far from what the data may suggest, which may cause bias in the model fit analysis. For our study, we model the observed data using a multivariate normal distribution, estimating each unique element of the mean vector and covariance matrix without constraints. For prior distributions, we specify a diffuse, uninformative prior of multivariate normal distribution for the mean vector with zero mean vector, zero-off diagonal elements of the prior covariance matrix and variances set to 100,0000 . For the saturated model covariance matrix $\boldsymbol{\Sigma}_{1}$, we also specify a diffuse, uninformative prior using an inverse Wishart prior distribution with parameters $\Psi$ 
with zero-off diagonal elements of item variances and degree of freedom $\boldsymbol{\nu}$ equals number of indicator variables.

Following estimation and successful convergence of the saturated model, the posterior predictive distributions of each of the means and covariances of the saturated model are formed using the typical PPMC process of sampling draws from the posterior distribution, using those parameters to generate simulated data $\boldsymbol{Y}^{\text {rep }}$, and calculating the Pearson correlation to every item pair, forming $T\left(\boldsymbol{Y}^{\text {rep }}\right)$. We then quantify the overlap between the alternative posterior predictive distribution with the reference posterior predictive distribution considered as a fully Bayesian analog of a traditional p-value.

If the model is consistent with the population that generated the observed data, then the posterior predictive distributions should have considerable overlap. A nonparametric test of the equality of probability distributions, the Kolmogorov-Smirnov statistic (KS) is used to assess the distance between the current model with the saturated model. The PPMC-KS statistic is the maximum difference between the cumulative densities of the posterior predictive distribution of the specified model $\left(H_{0}\right)$ and the saturated model $H_{1}$ across the space of the test statistic (the Pearson correlation). Thus, a PPMC-KS value is obtained for each pair of observed indicator variables. Next, we test our new PPMC methods via a simulation study.

\section{Monte Carlo Simulation Study}

In this section, we report results from a simulation study designed to investigate the performance of MC-PPMC. Our study borrows simulation specifics from Hoofs et al. (2018). Data were generated using either one or two latent variables. For data generated with one latent variable, the correct model (the one-factor model) was then estimated and compared with an overspecified model (a two-factor model where equal numbers of items loaded onto both factors) and the saturated model using the PPMC-KS statistic. When data were generated based on a two-factor model, the correct model (a two-factor model) 
was then tested against one underspecified model (a one-factor model), two incorrectly specified models, and the saturated model (see Figure 1).

\section{Data Generation Methods}

The simulated data sets were generated based on three main experimental factors: (1) number of latent factors (i.e., one-factor structure - Model A0 and two-factor structure - Model B0; see Figure 1), (2) number of observed indicators (6 items or 12 items), and (3) sample size $(25,500$, and 2000) for a total of 12 conditions.

For the one-factor model, to mimic real data, the magnitude of the factor loadings were fixed to $0.4 / 0.6 / 0.8$. When 6 -item tests were generated with a one-factor structure (Model A0), the factor loadings for all items were set as follows $\lambda_{1}=\lambda_{2}=0.4$, $\lambda_{3}=\lambda_{4}=0.6, \lambda_{5}=\lambda_{6}=0.8$. The factor variance was set to 1 . The residual variances of the indicators are set as follows $\psi_{1}=\psi_{2}=0.84, \psi_{3}=\psi_{4}=0.64, \psi_{5}=\psi_{6}=0.36$. These values were picked to achieve observed indicator variables with varying levels of information about the latent trait. Similarly, when the population data matrix was generated based on 12 items and one factor (not shown in Figure 1), the factor loadings are set so that the first four items had a loading of 0.4 , the second four items had a loading of 0.6 , and the last four items had a loading of 0.8 . Both factor variances are set to 1 while the factor covariance is set to $\phi_{12}=0.4$. The residual variances of the indicators are set as $0.84 / 0.64 / 0.36$.

For the two-factor model, when data were generated with 6 items (see Figure 1 Model B0), the factor loadings were set as $\lambda_{1}=\lambda_{2}=\lambda_{3}=0.4, \lambda_{4}=\lambda_{5}=\lambda_{6}=0.8$. When data were generated with 12 items, the factor loadings were set as $\lambda_{1}=\ldots=\lambda_{3}=0.4$, $\lambda_{4}=\ldots=\lambda_{6}=0.8, \lambda_{7}=\ldots=\lambda_{9}=0.4$, and $\lambda_{10}=\ldots=\lambda_{12}=0.8$. There were no cross-loadings for all models used for simulating data. For all two-factor models, the factor covariance is set to $\phi_{12}=0.4$. The residual variances of the indicators are set as 0.84 for item 1 to 3, 0.64 for item 4 to $6,0.35$ for item 7 to 9 .

The item intercepts $\boldsymbol{\mu}=\left(\mu_{1}, \ldots, \mu_{i}\right)^{T}$ for all generated data are fixed to 0 . Item 
response data were generated using factorial analytic equations given by Equation (1). The Bayesian estimation process used in each condition is explained in detail in the next section.

\section{Simulation Design}

In this section, we show the factor structure of the misspecificed model and the choices for prior distributions used in this study. In the first condition (data generated with a one-factor model), we analyzed the data with the saturated model and the misspecified two-factor model (Figure 1, Model B1: half of the items load onto the first factor and half of the items load onto the second factor). In the second condition, we estimated the model with three types of misspecification and the true model for comparison. As shown in Figure 1, the incorrect model B1 has one latent factor. The incorrect model B2 has one incorrect loading $\lambda_{1}$ for item 1 . The incorrect model B3 has the correct number of dimensions but has one item with an additional, unnecessary factor loading, $\lambda_{c}$.

Each condition was replicated 100 times. All models were estimated using MCMC estimation via JAGS (Plummer, 2003) with uninformative priors. Specifically, we set the prior distribution of factor loadings using a normal distribution $(\mu=0, S D=1)$. The prior distribution of item means was set to be a normal distribution with mean zero and variance of 100,000; the unique variances were sampled from a gamma prior distribution with alpha of .5 and beta of .059 ; the factor variance matrix in three-factor model was sampled from inverse Wishart distribution with $\Psi$ as a identity matrix and degree of freedom of 3. Each MCMC analysis had 4 estimation chains with 5,000 iterations of which 2,000 iterations were discarded as a burn-in phase. Following the analysis, 1,000 sets of parameters were then randomly drawn from the posterior distribution of MCMC estimates to generate posterior predictive data sets. To examine local misfit, for each pair of observed indicators, the PPMC-KS statistic was calculated comparing the posterior predictive distributions between a given model (correct or misspecified) and the saturated model.

In order to compare with Bayesian estimation, we also fit the models using 
maximum likelihood estimation using the Lavaan package (Rosseel, 2012) in $\mathrm{R}$ version 4.0 (R Core Team, 2020).

\section{Results}

\section{Global Model Fit}

Before comparing the proposed method, we checked the global model fit to investigate the overall model-data misfit. Table 1 shows the average values and the rejection rates of four global model fit indices (SRMR, CFI, TLI and RMSEA) across all 18 conditions (6 models by 3 sample sizes). The results suggested that when the true model has a one-factor structure, model A1 has the lowest SRMR/RMSEA and higher average CFI/TLI than model A0 because of overspecification. Similar to that, when the true model has a two-factor structure, model B0 and model B3 has lower SRMR/RMSEA and higher CFI/TLI than model B1 and B2. It should be noted that the model B3 has almost same global model fit as Model B0. It is not surprising because model B3 has only one more cross-loading than model B0.

As for the influence of sample sizes, as sample size increases, all fit indices indicate better model fit when the true model is following either one-factor structure or two-factor structure. Both model A0 and A1 have good model fit when sample sizes are larger than 25. Models B1 and B2 have poor model fit uniformly, even when the sample size is 2,000. Model B0 and model B3 have acceptable model fit when sample size larger than 50.

\section{PPMC-KS and PPP values}

To illustrate the differences between PPMC-KS measures and PPP values, we re-emphasize that PPMC-KS statistics and PPP values have distinct criteria for misfit. When the PPMC-KS statistic of an item pair correlation is close to zero, the posterior predictive distribution of the discrepancy measure in the alternative model completely overlaps with that of the measure in the saturated model, meaning near perfect model-data 
fit. Similarly, if the PPMC-KS is close to one or the PPP value is close to either zero or one, local misfit is present. To provide more direct comparisons, we transformed the PPP values to the an absolute PPP (Equation 5) so that lower absolute PPP values suggest better local fit.

$$
\mathrm{PPP}_{\text {new }}=|P P P-0.5|
$$

Figure 2 shows how PPMC-KS (red solid line) and $\mathrm{PPP}_{\text {new }}$ values (blue dashed line) perform differently for the same six-item test. The left-hand side panel shows the average $\mathrm{PPP}_{\text {new }}$ values and PPMC-KS statistics across all 100 replications for each observed indicator correlation in the one-factor solution (Model A0). The right-hand side panel of Figure 2 shows the results in the two-factor solution (Model A1). The results suggest that the $\mathrm{PPP}_{\text {new }}$ values and PPMC-KS have similar trends when sample size increases. The two indices suggest different local model-data fit between two solutions. Specifically, for item pair correlations between indicators both loading onto the same factor (i.e., indicators 1 and 2, indicators 1 and 3), the PPMC-KS and the PPP new values are not affected by small sample size in the one-factor solution (correct model, Model A0). In contrast, in the two-factor solution (Model A1), the PPMC-KS and the $\mathrm{PPP}_{\text {new }}$ values increase for some correlations (i.e., indicators 1 and 2, indicators 1 and 3) as the sample size gets larger. For $N=2000,6$ out of 15 correlations in the overspecified model have higher $\mathrm{PPP}_{\text {new }}$ values than in the correct model; 12 out of 15 correlations in the overspecified model have higher PPMC-KS statistics than in the correct model. For $N=25$, only two correlations are flagged as higher $\mathrm{PPP}_{\text {new }}$ values in the overspecified model than in correct model whereas five correlations have higher PPMC-KS statistics.

The results are consistent for the 12-item two-factor condition (Model B0) and other factor structures. Figure 3 shows the PPMC-KS and $\mathrm{PPP}_{\text {new }}$ for six item pair correlations with the 12-item test in four solutions. Here, the $\mathrm{PPP}_{\text {new }}$ values (blue dashed line) in three sample size conditions have similar patterns with the PPMC-KS statistics (red solid line). It should be noted that the correct model has the best model fit overall 
(lowest $\mathrm{PPP}_{\text {new }}$ values and PPMC-KS statistics). Even for small sample sizes $(N=25)$, the $\mathrm{PPP}_{\text {new }}$ values and the PPMC-KS statistics for Model B0 show good performance. For comparison, the underspecified model (Model B1) has relatively higher $\mathrm{PPP}_{\text {new }}$ values and PPMC-KS statistics for item pairs with two observed indicators loading onto different factors. The incorrect model with one observed indicator loading onto the wrong factor (Model B2) also has large $\mathrm{PPP}_{\text {new }}$ values and PPMC-KS statistics for all pairs that including observed indicator 1. Similarly, the incorrect model with observed indicator 1 cross-loading on two factors (Model B3) has large PPP ${ }_{\text {new }}$ values and PPMC-KS statistics for observed indicator pair that includes item 1.

Even though the $\mathrm{PPP}_{n e w}$ values and PPMC-KS statistics have similar patterns, there are some differences in their sensitivity. For example, in Model B2 (overspecified model), the $\mathrm{PPP}_{\text {new }}$ values of observed indicator pairs 1 with 10, 11, and 12 are close to their upper threshold which indicates overestimation when sample size larger than 500. $\mathrm{PPP}_{\text {new }}$ values of observed indicator pairs 1 with 4, 5, and 6 suggest the underestimation of those correlations. However, the PPMC-KS for the same indicator pairs (indicators 1 and 10, 1 and 11, and 1 and 12) did not reach the upper threshold. This result may suggest that PPMC-KS statistics are sensitive to overestimation but less sensitive to underestimation. Finally, comparing $\mathrm{PPP}_{\text {new }}$ values to PPMC-KS statistics, it seems that standard PPP values larger than .95 or lower than .05 corresponds to PPMC-KS statistics larger than .5. Thus, .5 may be a fair cut score for the PPMC-KS approach.

\section{Empirical Data Analysis}

\section{Method}

In this section, we illustrate how the proposed PPMC approach could be used to obtain better model fit and select better model when using Bayesian confirmatory factor analysis. This section does not provide, however, a comprehensive overview of an actual Bayesian CFA analysis. The goal of the empirical illustration was to demonstrate how the 
researchers could detect the local misfit of BCFA models or compare the models when multiple alternative models exist.

Data from Holzinger and Swineford (1939) were used. Test scores on 26 different measures were obtained from a total of 3007 th and 8th grade students in two schools. The Holzinger and Swineford (1939) data have been used as a model data set by many investigators. For example, Muthén and Asparouhov (2012) used the factor loading pattern of the four-factor model as Table 2 shown. To be specific, 19 out of 26 items were intended to measure four correlated latent factors: (1) spatial $\left(\eta_{1}\right)$ measured by visual perception, cubes, paper form board, and flags $\left(x_{1}-x_{4}\right),(2)$ verbal $\left(\eta_{2}\right)$ measured by general information, paragraph comprehension, sentence completion, word classification, and word meaning $\left(x_{5}-x_{9}\right)$, (3) speed $\left(\eta_{3}\right)$ measured by addition, code, counting groups of dots, and straight and curved capitals $\left(x_{10}-x_{13}\right)$, and (4) memory $\left(\eta_{4}\right)$ measured by word recognition, number recognition, figure word $\left(x_{14}-x_{19}\right)$. To illustrate the performance of the proposed method, two models are estimated: a one-factor model and a four-factor model (Asparouhov \& Muthén, 2020). The detailed specification for the four-factor solution is shown in Table 2, All test scores were standardized before the analysis.

The data were estimated with one-factor structure and four-factor structure using BSEM via MCMC estimation. The process includes 4 chains with 10,000 iterations in which 2,000 are discarded as burn-in. The prior settings for the Bayesian estimation are as follows: item intercepts are normally distributed with mean 0 and variance 1; factor loadings are normally distributed with mean 0 and variance 1 . For model identification, both one-factor solution and four-factor solution employ the marker-item method, which mean the factor loadings of first item per factor are fixed to 1 while other factor loadings are freely estimated. After the posterior distributions for all parameters were estimated, 5,000 parameters from the posterior were randomly sampled and used to create the posterior predictive distribution for the 271 item-pair correlations. 


\section{Results}

Using the Gelman-Rubin convergence diagnostic, both the one-factor solution $(R \leq 1.004)$ and four-factor solution $(R \leq 1.033)$ achieved convergence. Figure 4 presents the distribution of PPMC-KS statistics and $\mathrm{PPP}_{\text {new }}$ values across all item-pair correlations with the one-factor solution and the four-factor solution. As shown in the boxplot, the average PPMC-KS statistics are higher in the one-factor model $\left(\mu_{P P M C-K S}=.446\right)$ than in the four-factor model $\left(\mu_{P P M C-K S}=.310\right)$, which indicates worse local model fit in the one-factor solution than in the four-factor solution. In addition, the range and standard deviation of the PPMC-KS statistics in the one-factor model $(s d=.319)$ are wider than in the four-factor model $(s d=.198)$, which indicates the higher variation of PPMC-KS statistics in the one-factor model. From the one-factor model results, the highest PPMC-KS statistic was found in the correlation between indicator pair 10 and 12 $(\mathrm{PPMC}-\mathrm{KS}=.999)$ while in four-factor model, the highest KS statistics was found in the correlation between indicator pair 2 and $10(\mathrm{PPMC}-\mathrm{KS}=.837)$. These results suggest that according to the PPMC-KS results, the four-factor model fixes much of the local misfit in one-factor model.

For comparison, Figure 5 shows the distribution of PPP values across the whole item-pair correlations with the one-factor and four-factor models. Here, similar to the PPMC-KS statistics, the average $P P P$ value for the four-factor model is closer to .5 $(P \bar{P} P=.525)$ than the one-factor model $(P \bar{P} P=.699)$. However, the range and standard deviation for the one-factor $(s d=.297)$ and four-factor models $(s d=.302)$ are very similar. In addition, in the one-factor model, 25 item-pair correlations yielded $P P P$ values equal to one. One of the problematic item-pair correlations included the correlation between items 10 and 12, which also had the highest PPMC-KS statistic. Indicator pair 2 and 10 had the lowest $P P P$ value $(P \bar{P} P=.021)$ in the one-factor model. For the four-factor model, indicator pair 2 and 10 had the lowest $P P P$ value and indicator pair 8 and 10 had the highest $P P P$ value. 


\section{Discussion}

In this study, we proposed a model comparison approach to model checking in Bayesian CFA. In our investigation, we showed acceptable sensitivity of PPP values for three forms of misspecification (underspecified, overspecified, wrongly specified) when sample size is moderately large (i.e., $N=500$ ), in accordance with previous studies (e.g., Hoofs et al., 2018). However, similar to the findings of previous literature, PPP values were insensitive to small samples combined with an overspecified model. Our simulation study shows that the PPMC using KS statistics could be an alternative way of detecting local misfit in Bayesian CFA. For large sample sizes, PPMC-KS shows similar patterns with $\mathrm{PPP}_{\text {new }}$ values. When sample sizes are small, more indicator correlations show higher PPMC-KS statistics in the overspecified model than those in correct model; for comparison, less than half item correlations show higher $\mathrm{PPP}_{\text {new }}$ values.

\section{PPMC using Saturated model vs. PPP value}

In summary, there are several similarities between PPMC-KS statistics method and PPP values in this data analysis. First, both PPMC-KS statistics and PPP methods suggest more local misfit exists in one-factor models than in four-factor models, which is consistent with previous literature (Asparouhov \& Muthén, 2020). Second, both PPMC-KS statistics and PPP values indicate the same largest local misfit, which is indicator pair 10 with 12 in the one-factor model and indicator pair 2 with 10 in the four-factor model. However, there are also some differences between these two approaches. Using PPP values, some indicator pair correlations may reach the maximum of one (i.e., 25 item pair correlations have a PPP value 1 in the one-factor model) but the PPMC-KS statistics were never as extreme which makes model comparison possible.

The PPMC approach using a saturated model could be a very useful tool for detecting local misfit in a fully Bayesian framework. The underlying idea of comparing a saturated model to an alternate model is consistent with the ML-based model fit (i.e. 
RMSEA, SRMR). The only difference is that the model-data fit is represented by the overlap between the posterior predictive distribution of test statistics rather than a chi-square difference.

Both the PPP approach and PPMC-KS statistics are good ways for checking local misfit in Bayesian CFA. When fit is poor, PPMC-KS statistics may be more informative than PPP as PPMC-KS statistics never reach extreme values. In summary, PPMC-KS statistics could be a supplementary approach for PPP methods for checking local misfit in Bayesian CFA.

This study has a few limitations which can be considered as future research directions. One limitation of this study is missing the cutoff scores for the PPMC-KS statistic. The universal cutoff scores for PPMC-KS statistics may not exist but false discovery rate - a method to control error rate could be further investigated to find the criterion for PPMC-KS statistics. 


\section{References}

Asparouhov, T., \& Muthén, B. (2020). Advances in Bayesian Model Fit Evaluation for Structural Equation Models. Structural Equation Modeling: A Multidisciplinary Journal, 1-14. https://doi.org/10.1080/10705511.2020.1764360

Boomsma, A. (1987). The Robustness of Maximum Likelihood Estimation in Structural Equation Models. Structural modeling by example: Applications in educational, sociological, and behavioral research (pp. 160-188). Cambridge University Press.

Garnier-Villarreal, M., \& Jorgensen, T. D. (2019). Adapting Fit Indices for Bayesian Structural Equation Modeling: Comparison to Maximum Likelihood. Psychological Methods, No Pagination Specified-No Pagination Specified. https://doi.org/10.1037/met0000224

Gelman, A., Meng, X.-L., \& Stern, H. (1996). Posterior Predictive Assessment of Model Fitness Via Realized Discrepancies. Statistica Sinica, 733-807.

Hoofs, H., van de Schoot, R., Jansen, N. W. H., \& Kant, I. (2018). Evaluating Model Fit in Bayesian Confirmatory Factor Analysis With Large Samples: Simulation Study Introducing the BRMSEA. Educational and Psychological Measurement, 78(4), 537-568. https://doi.org/10.1177/0013164417709314

Kaplan, D. (1988). The Impact of Specification Error on the Estimation, Testing, and Improvement of Structural Equation Models. Multivariate Behavioral Research, $23(1), 69-86$.

Lee, T., Cai, L., \& Kuhfeld, M. (2016). A Poor Person's Posterior Predictive Checking of Structural Equation Models. Structural Equation Modeling: A Multidisciplinary Journal, 23(2), 206-220. https://doi.org/10.1080/10705511.2015.1014041 _eprint: https://doi.org/10.1080/10705511.2015.1014041

Levy, R. (2011). Bayesian Data-Model Fit Assessment for Structural Equation Modeling. Structural Equation Modeling: A Multidisciplinary Journal, 18(4), 663-685. https://doi.org/10.1080/10705511.2011.607723 
Levy, R., Mislevy, R. J., \& Sinharay, S. (2009). Posterior Predictive Model Checking for Multidimensionality in Item Response Theory. Applied Psychological Measurement, $33(7), 519-537$.

McDonald, R. P. (1999). Test theory: A unified approach. Mahwah, NJ: Erlbaum.

Muthén, B., \& Asparouhov, T. (2012). Bayesian structural equation modeling: A more flexible representation of substantive theory. Psychological Methods, 17(3), 313-335. https://doi.org/10.1037/a0026802

Plummer, M. (2003). JAGS: A program for analysis of Bayesian graphical models using Gibbs sampling.

R Core Team. (2020). R: A language and environment for statistical computing. $\mathrm{R}$ Foundation for Statistical Computing. Vienna, Austria. https://www.R-project.org/

Robins, J. M., van der Vaart, A., \& Ventura, V. (2000). Asymptotic Distribution of P Values in Composite Null Models [_eprint: https://doi.org/10.1080/01621459.2000.10474310]. Journal of the American Statistical Association, 95(452), 1143-1156. https://doi.org/10.1080/01621459.2000.10474310

Rosseel, Y. (2012). Lavaan: An R package for structural equation modeling and more. Version 0.5-12 (BETA). Journal of statistical software, 48(2), 1-36. 


\section{Table 1}

Comparing Global Fit Indices: Average Values and Rejection Rates for ML estimators.

\begin{tabular}{cccccc}
\hline Model & $N$ & SRMR & CFI & TLI & RMSEA \\
\hline A0 & 50 & $.033 / .060$ & $.989 / .020$ & $.991 / .055$ & $.046 / .410$ \\
A0 & 500 & $.010 / .000$ & $.999 / .000$ & $1.000 / .000$ & $.011 / .000$ \\
A0 & 2000 & $.005 / .000$ & $1.000 / .000$ & $1.000 / .000$ & $.006 / .000$ \\
A1 & 50 & $.032 / .045$ & $.990 / .020$ & $.992 / .060$ & $.045 / .390$ \\
A1 & 500 & $.010 / .000$ & $.999 / .000$ & $1.000 / .000$ & $.010 / .000$ \\
A1 & 2000 & $.005 / .000$ & $1.000 / .000$ & $1.000 / .000$ & $.006 / .000$ \\
B0 & 50 & $.065 / .885$ & $.965 / .250$ & $.962 / .335$ & $.063 / .640$ \\
B0 & 500 & $.020 / .000$ & $.999 / .000$ & $1.000 / .000$ & $.007 / .000$ \\
B0 & 2000 & $.010 / .000$ & $1.000 / .000$ & $1.000 / .000$ & $.003 / .000$ \\
B1 & 50 & $.197 / 1.000$ & $.635 / 1.000$ & $.578 / 1.000$ & $.235 / 1.000$ \\
B1 & 500 & $.188 / 1.000$ & $.664 / 1.000$ & $.612 / 1.000$ & $.221 / 1.000$ \\
B1 & 2000 & $.176 / 1.000$ & $.675 / 1.000$ & $.625 / 1.000$ & $.215 / 1.000$ \\
B2 & 50 & $.115 / .995$ & $.922 / .760$ & $.909 / .805$ & $.107 / .950$ \\
B2 & 500 & $.095 / .975$ & $.955 / .280$ & $.946 / .370$ & $.080 / .575$ \\
B2 & 2000 & $.091 / .932$ & $.956 / .304$ & $.947 / .372$ & $.078 / .595$ \\
B3 & 50 & $.063 / .830$ & $.965 / .250$ & $.962 / .340$ & $.063 / .645$ \\
B3 & 500 & $.020 / .000$ & $.999 / .000$ & $1.000 / .000$ & $.007 / .000$ \\
B3 & 2000 & $.010 / .000$ & $1.000 / .000$ & $1.000 / .000$ & $.003 / .000$ \\
\hline
\end{tabular}

Note: The values before the slash represent the average model fit; the value after the slash represent the proportion of models having unacceptable model fit among all repetitions. 


\section{Table 2}

Factor Structure of the Holzinger-Swineford example: Four-factor solution

\begin{tabular}{|c|c|c|c|c|}
\hline & Spatial & Verbal & Speed & Memory \\
\hline visual & $\mathrm{X}$ & 0 & 0 & 0 \\
\hline cubes & $\mathrm{X}$ & 0 & 0 & 0 \\
\hline paper & $\mathrm{X}$ & 0 & 0 & 0 \\
\hline flags & $\mathrm{X}$ & 0 & 0 & 0 \\
\hline general & 0 & $\mathrm{X}$ & 0 & 0 \\
\hline paragrap & 0 & $\mathrm{X}$ & 0 & 0 \\
\hline sentence & 0 & X & 0 & 0 \\
\hline wordc & 0 & $\mathrm{X}$ & 0 & 0 \\
\hline wordm & 0 & $\mathrm{X}$ & 0 & 0 \\
\hline addition & 0 & 0 & $\mathrm{X}$ & 0 \\
\hline code & 0 & 0 & $\mathrm{X}$ & 0 \\
\hline counting & 0 & 0 & $\mathrm{X}$ & 0 \\
\hline straight & 0 & 0 & X & 0 \\
\hline wordr & 0 & 0 & 0 & $\mathrm{X}$ \\
\hline numberr & 0 & 0 & 0 & $\mathrm{X}$ \\
\hline figurer & 0 & 0 & 0 & $\mathrm{X}$ \\
\hline object & 0 & 0 & 0 & $\mathrm{X}$ \\
\hline numberf & 0 & 0 & 0 & $\mathrm{X}$ \\
\hline figurew & 0 & 0 & 0 & $X$ \\
\hline
\end{tabular}




\section{Figure 1}

Simulation Design: Different Models

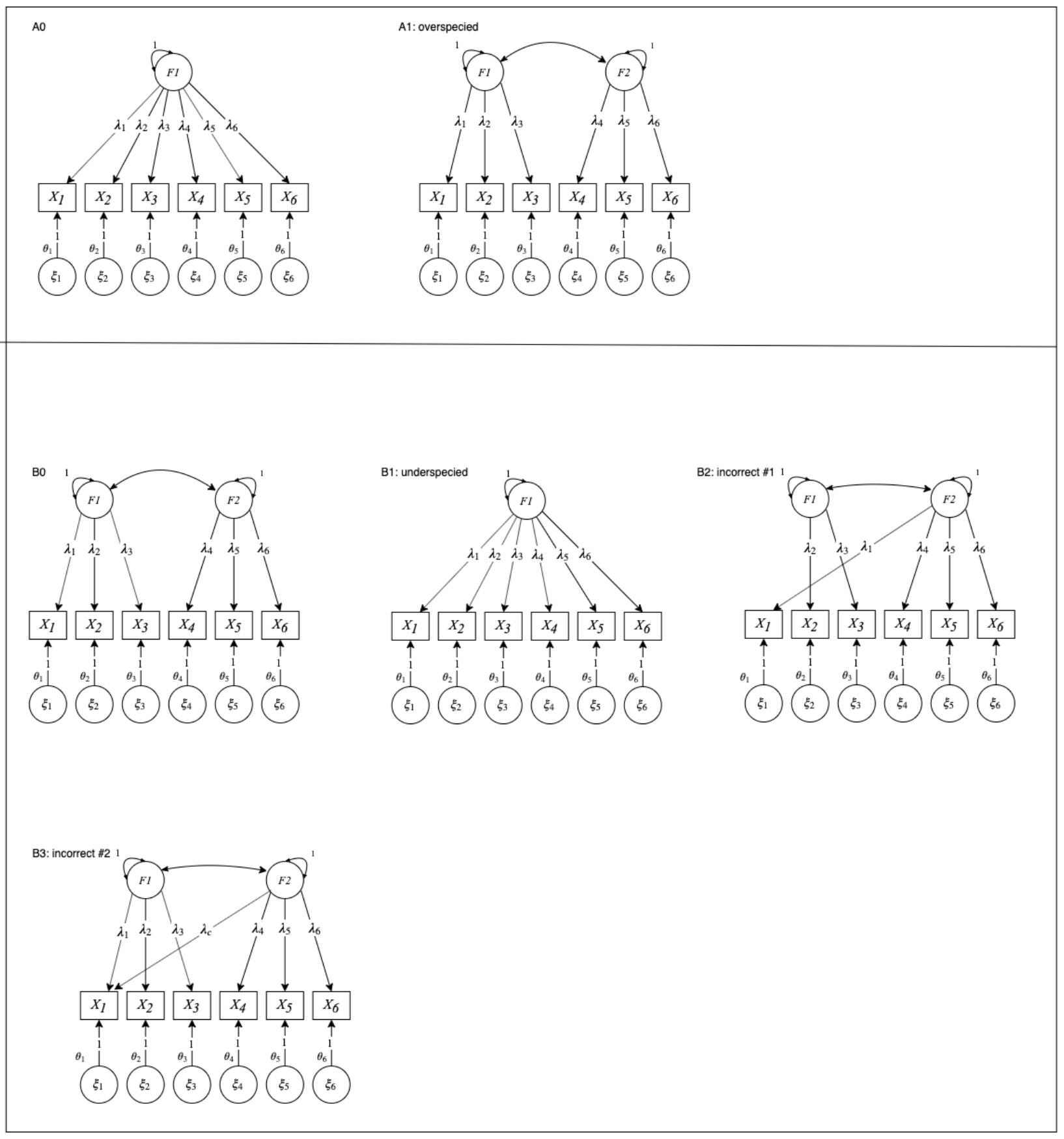


Figure 2

PPP Values and PPMC KS statistics in condition of 6-item with one-factor structure

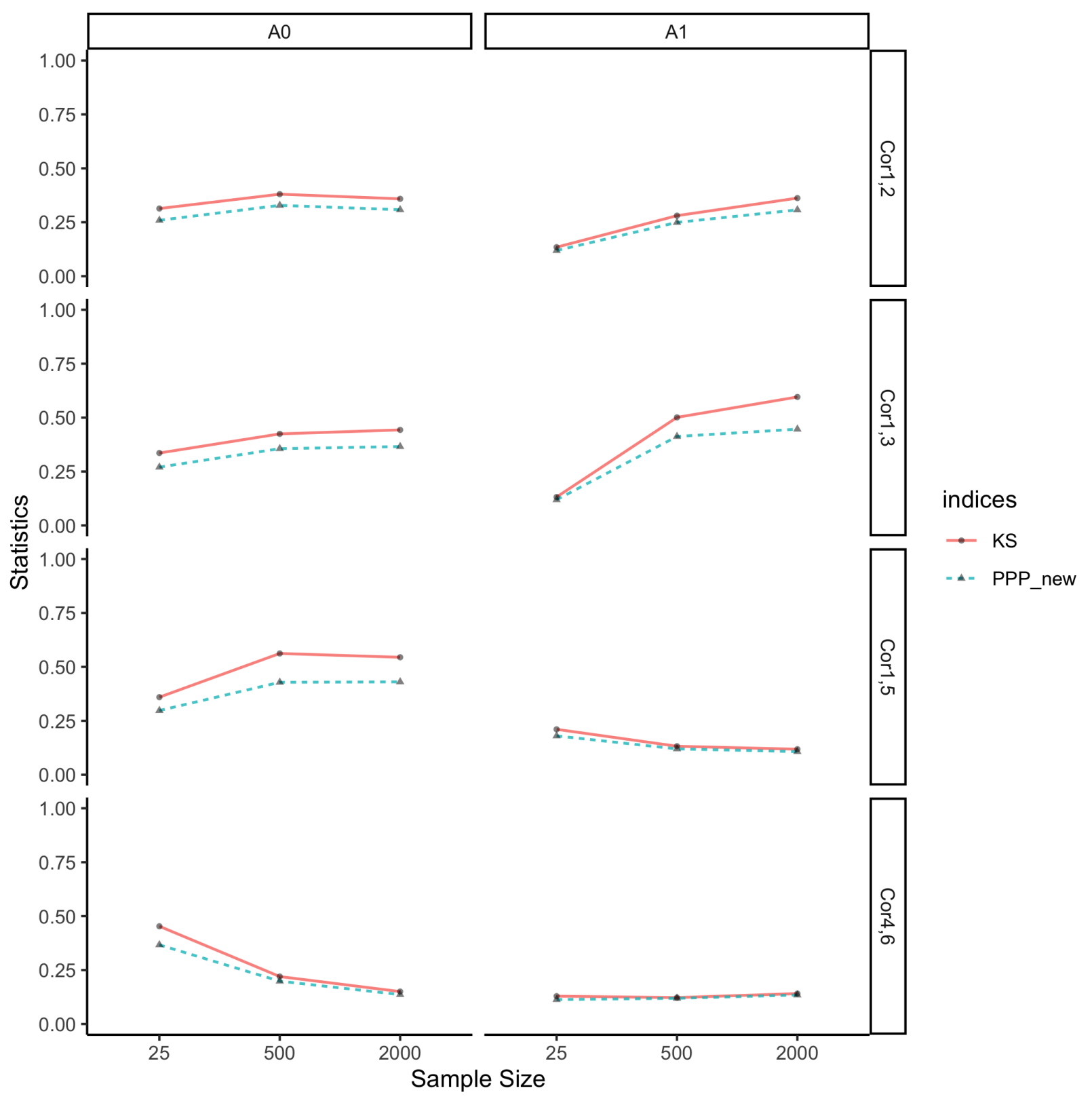



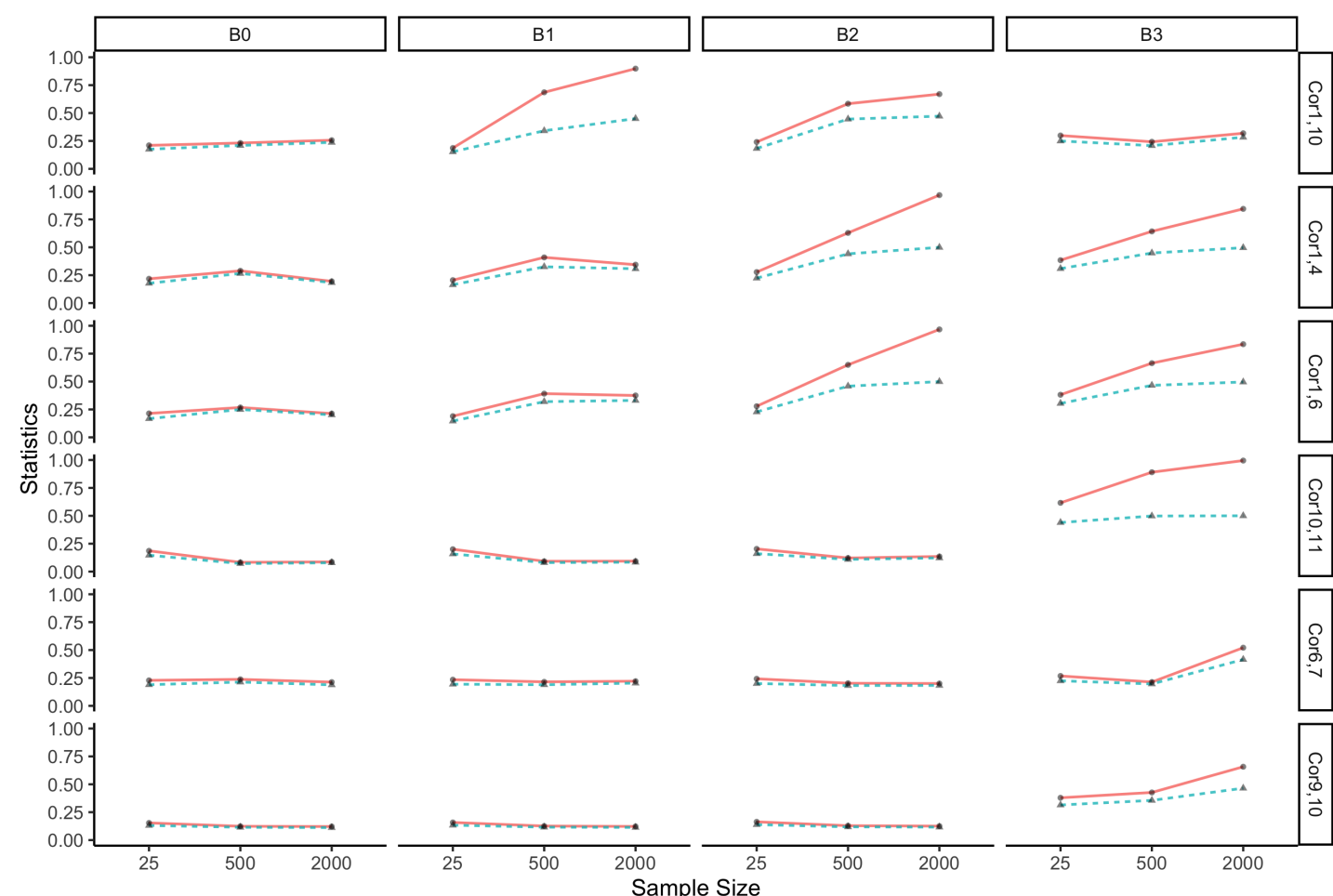

- - PPP_new

Figure 3

PPP Values and KS statistics: Model B0, B1, B2, B3 
Figure 4

KS Statistics Boxplot for one-factor and four-factor solutions

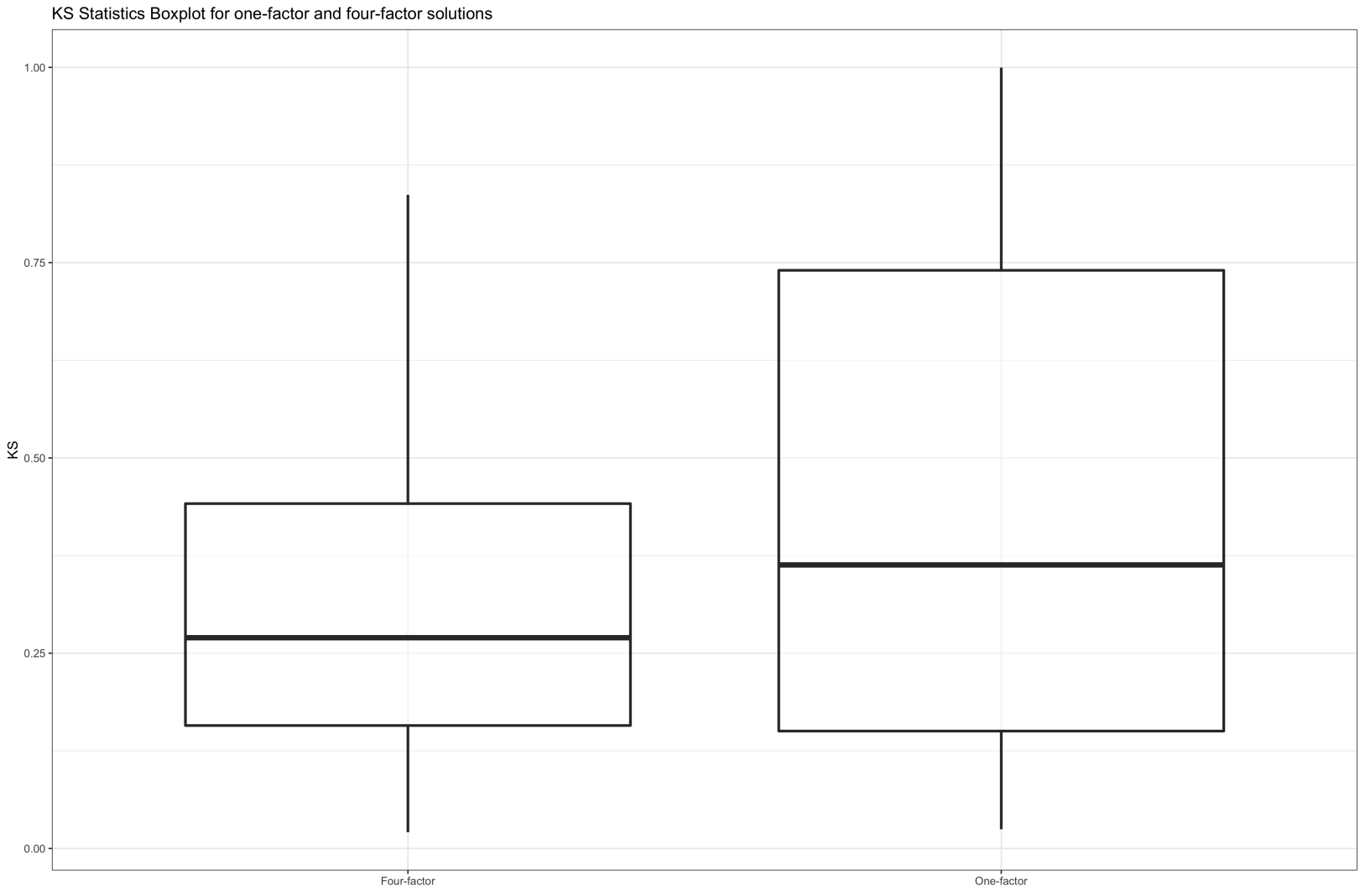




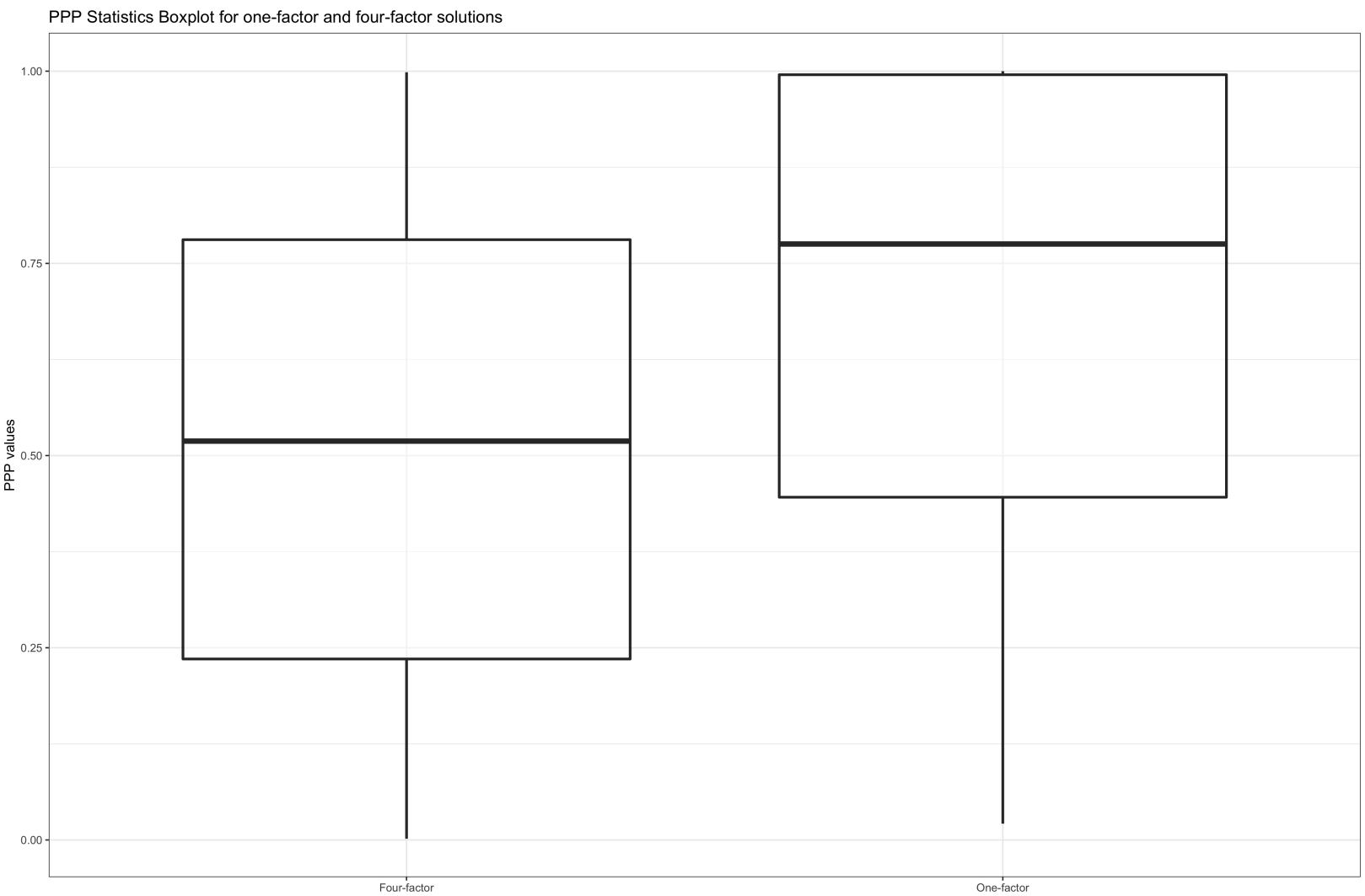

Figure 5

PPP Statistics Boxplot for one-factor and four-factor solutions 